\title{
On characterizing skeletal muscle contraction properties. Experimental and simulation methodology
}

\author{
M. Sierra1, M.J. Muñoz1, J. Grasa2 \\ 1 Laboratorio de Genética Bioquímica (Lagenbio) \\ Instituto de Investigación en Ingeniería de Aragón (I3A). \\ Universidad de Zaragoza, Mariano Esquillor s/n, 50018, Zaragoza, Spain. \\ Tel. +34-976762707, Fax +34-976762043 \\ 2 Grupo de Mecánica Aplicada y Bioingeniería (AMB) \\ Instituto de Investigación en Ingeniería de Aragón (I3A). \\ Universidad de Zaragoza, Mariano Esquillor s/n, 50018, Zaragoza, Spain. \\ Tel. +34-976762707, Fax +34-976762043
}

\begin{abstract}
The main objective of this work is to characterize the fatigue contractile properties of three different rat muscles (Tibialis Anterior, Extensor Digitorium Longus and Soleus) in order to obtain experimental parameters for numerical simulations.

Experiments were conducted "in vivo" on three groups $(\mathrm{n}=6)$ of male Wistar rats $(210+/-11 \mathrm{~g})$ using a protocol developed by authors in previous works. Muscles were subjected to an electrical stimulus to achieve tetanic contraction during ten seconds. Digital Image Correlation was used during tests for 3D strain and displacements measurement that allow the correlation with the finite element simulations.

By means of Computed Tomography, a precise reconstruction of both bone and muscle of the rat hindlimb geometry was obtained. The methodology proposed allows to obtain and validate computational simulations of skeletal muscle fatigue under different characteristics related to fiber types.
\end{abstract}

